\title{
ESTUDO DA COERÊNCIA DO ELETRENCEFALOGRAMA PARA A BANDA DE FREQUÊNCIA ALFA EM INDIVÍDUOS ADULTOS NORMAIS E COM PROVÁVEL DEMÊNCIA DO TIPO ALZHEIMER
}

\author{
RENATO ANGHINAH*, PAULOAFONSO MEDEIROS KANDA*, MÁRIO SILVA JORGE*, \\ EDSON ERASMO PEREIRA DE LIMA**, LUIZPASCUZZI***, ANTÔNIO CARLOS DE PAIVA MELO****
}

RESUMO - Estudamos a coerência inter-hemisférica do espectro do eletrencefalograma (EEG) obtido pela transformada rápida de Fourier, da região occipital do escalpo (eletrodos $\mathrm{O} 1$ e O2) para a banda de frequência alfa (alfa $-8,0$ a $10,0 \mathrm{~Hz}$ e alfa $_{2}-10,1 \mathrm{a} 12,5 \mathrm{~Hz}$ ) em indivíduos normais maiores de 50 anos e pacientes com diagnóstico provável de demência do tipo Alzheimer (DTA), no intuíto de observar se há diferenças entre estes parâmetros que contribuam com o diagnóstico desta enfermidade. Encontramos diminuição na coerência interhemisférica dos indivíduos com DTA para ambas as sub-bandas do ritmo alfa. Acreditamos que a análise de coerência do EEG possa ser um método de grande auxílio no diagnóstico de DTA.

PALAVRAS-CHAVE: eletrencefalograma quantitativo, análise de coerência, demência.

\section{Alpha band coherence analysis of EEG in healthy adult and Alzheimer's type dementia subjects}

ABSTRACT - We studied the occiptal inter-hemispheric coherence (IHCoh) of EEG (electrodes O1-O2) for alpha band (alpha $-8,0$ to $10,0 \mathrm{~Hz}$ and alpha $_{2}-10,1$ to $12,5 \mathrm{~Hz}$ ) in healthy adults and Alzheimer's type dementia (ATD) subjects, to observe if there is any significant difference between these two groups that could help in the early diagnosis of ATD. We found a decrease of occipital IHCoh in ATD group for both alpha sub-bands. We believe that Coh analysis of EEG is a powerful auxiliary method in ATD diagnosis.

KEY WORDS: quantitative electroencephalography, coherence analysis, dementia.

Coerência (Coh) é a medida da covariância do espectro de dois sinais de eletrencefalograma (EEG). A alta Coh entre dois sinais do EEG tem sido considerada como evidência estrutural e funcional da ocorrência de conexões entre áreas corticais ${ }^{1}$. Desde os primeiros estudos da quantificação do EEG por Lehmann $(1971)^{2}$ e Duffy e cols.. (1979) ${ }^{3}$ o estudo da análise espectral e estatística do EEG vem sendo largamente utilizado. Contrariamente a isto, estudos da Coh do EEG são raramente encontrados na literatura ${ }^{4}$. A informação obtida pelo estudo da Coh é essencial para o entendimento de como relacionamentos mútuos entre regiões cerebrais podem mudar sob diferentes condições ${ }^{5}$.

Das infinitas possibilidades de correlações entre diferentes áreas cerebrais, a análise de Coh é uma medida já estabelecida como capaz de mensurar as relações inter- hemisféricas através do corpo caloso tanto na vigília como no sono ${ }^{6}$.

*Médico pós-graduando do Setor de Eletroencefalografia (EEG) do Hospital do Servidor Público do Estado (HSPE); **Neuropsicólogo do Ambulatório de Funções Corticais do HSPE; **** Médico Chefe do Setor de EEG do HSPE; *****ádico Diretor do Serviço de Neurologia do HSPE. Aceite: 13-dezembro-1999.

Dr. Renato Anghinah - Rua Itacolomi 333/83 - 01239020 São Paulo SP - Brasil. E-mail: anghinah@opus.com.br 
A Análise de Coh do EEG tem sido empregada de maneira experimental no estudo das demências. Leuchter e col. ${ }^{7}$ estudaram pacientes com demência tipo Alzheimer (DTA) e demência tipo multi-infartos (DMI) e encontraram coerência menor em pacientes com DMI em relação aos DTA. Porém, não encontraram diferenças estatisticamente significantes entre o grupo DTA e o grupo controle de indivíduos normais ${ }^{8}$. Besthorn e col. ${ }^{5}$ estudaram um grupo de 50 pacientes com hipótese diagnóstica de DTA e compararam com grupo controle normal, encontrando coerência diminuída nas regiões centrais e frontais, achado semelhante também encontrado por Locatelli e col. ${ }^{9}$ cujos estudos mostraram coerência diminuída para a banda alfa na regiões temporo-parietooccipitais à esquerda ${ }^{5,9}$.

Estudos recentes mostram que há diminuição na coerência inter-hemisférica das regiões posteriores de pacientes com provável DTA, quando comparados a grupos controles normais ${ }^{6,10,11}$. Ao compararmos a sensibilidade da análise de coerência com outros métodos diagnósticos das demências alguns estudos mostraram forte correlação entre as alterações da coerência e testes neuropsicológicos ${ }^{12-}$ ${ }^{13}$. Já Sloam e col. ${ }^{14}$ mostraram achados congruentes entre a análise de coerência do EEG e pela tomografia por emissão de pósitron único (SPECT). Resultados semelhantes foram obtidos por Szelies e col. ${ }^{15}$ ao comparar EEG quantitativo (EEGQ) a tomografia por emissão de pósitrons (PET).

Nosso objetivo neste estudo foi comparar a Coh cerebral do EEG entre um grupo de indivíduos normais e outro com diagnóstico provável de DTA, no intuito de avaliar este método como um auxiliar no diagnóstico precoce desta enfermidade.

\section{MÉTODO}

Foram selecionados dois grupos de indivíduos: o primeiro (G1) composto por 5 indivíduos normais, de ambos os sexos e com idade superior a 50 anos, e o segundo (G2) composto por 11 individuos de ambos os sexos, com queixas de déficits de memória e/ou deterioração das funções intelectuais, e diagnóstico provável de DTA segundo avaliação neuropsicológica e mini-exame do estado mental (MEEM) com escores inferiores a 24 pontos, respeitando-se o nível de escolaridade ${ }^{16}$.

Excluímos do presente estudo indivíduos com antecedentes de diabetes mellitus, nefropatias, tireoideopatias, alcoolismo, hepatopatias, doenças pulmonares e carência de vitamina B12.

Os registros do EEG quantitativo foram realizados em equipamento digital da marca EMSA, com 20 canais e processador de 12 bits, capacitado a realizar estudos quantitativos do EEG e análise de Coh por transformada rápida de Fourier (FFT), utilizando a fórmula:

$$
\begin{aligned}
\text { Coh (f) } 2=\frac{\text { Gxy (f) } \mid 2}{\operatorname{Gxx}(f) G y y(f)} & ===>\text { Potência do espectro cruzado }
\end{aligned}
$$

A colocação dos eletrodos de escalpo seguiu as normas da Sociedade Brasileira de Neurofisiologia Clínica e da Sociedade Americana de Neurofisiologia Clínica ( sistema 10-20 com referência bi-auricular unida) para a aquisição do EEG ${ }^{17-19}$.

Estudamos a Coh inter-hemisférica entre os eletrodos O1-O2 dos grupos G1 e G2 para as sub-bandas alfa (alfa1 8,0 a 10,0 Hz e alfa 2 de 10,1 a $12,5 \mathrm{~Hz}$ ).

Submetemos os resultados à análise estatística pelo teste t para duas amostras, presumindo-se variâncias equivalentes.

\section{RESULTADOS}

Foram encontrados para o Grupo 1: banda alfa 1 Coh entre 0,59 e 0,75 com média de 0,688, e para alfa 2 valores entre 0,60 e 0,65 , com média de 0,628 (tabela 1 ).

Para o Grupo 2 encontramos: banda alfa 1, Coh entre 0,37 e 0,69 com média de 0,51; e para alfa 2 valores entre 0,31 e 0,56 , com média de 0,44 (tabela 2).

O cálculo estatístico $(Z)$ mostra significância estatística entre os grupos: coh alfa1 G1xG2 $(\mathrm{p}=0,0010)$; e alfa $2 \mathrm{G} 1 \times \mathrm{xG} 2(\mathrm{p}=0,00045)$. 
Tabela 1. Indivíduos adultos normais maiores de 50 anos.

\begin{tabular}{lcccc}
\hline Grupo I & $\begin{array}{c}\text { Idade } \\
\text { (anos) }\end{array}$ & MEEM & $\begin{array}{c}\text { Coh O1-2 } \\
\text { banda } \alpha 1\end{array}$ & $\begin{array}{c}\text { Coh O1-2 } \\
\text { banda } \alpha 1\end{array}$ \\
\hline IZC & 62 & 28 & 0,68 & 0,60 \\
RPM & 70 & 28 & 0,68 & 0,64 \\
ATT & 70 & 29 & 0,75 & 0,62 \\
JAN & 52 & 29 & 0,74 & 0,63 \\
LLG & 60 & 28 & 0,59 & 0,65 \\
Média & & & 0,688 & 0,628 \\
\hline
\end{tabular}

MEEM, mini exame do estado mental; Coh, coerência; O1-2, eletrodos occipitais.

Tabela 2. Pacientes adultos com DTA.

\begin{tabular}{lcccc}
\hline Grupo II & $\begin{array}{l}\text { Idade } \\
(\text { anos })\end{array}$ & MEEM & $\begin{array}{c}\text { Coh O1-2 } \\
\text { banda } \alpha 1\end{array}$ & $\begin{array}{r}\text { Coh O1-2 } \\
\text { banda } \alpha 1\end{array}$ \\
\hline GMA & 85 & 12 & 0,50 & 0,39 \\
AMP & 73 & 12 & 0,55 & 0,50 \\
MAM & 68 & 22 & 0,69 & 0,56 \\
AON & 63 & 21 & 0,44 & 0,31 \\
GPF & 90 & 22 & 0,59 & 0,42 \\
GOS & 78 & 23 & 0,42 & 0,41 \\
APL & 74 & 14 & 0,49 & 0,33 \\
JSB & 75 & Afásico & 0,37 & 0,37 \\
VCS & 59 & 17 & 0,57 & 0,56 \\
AS & 75 & 22 & 0,59 & 0,56 \\
MAF & 71 & 17 & 0,45 & 0,44 \\
Média & & & 0,51 & 0,44 \\
\hline
\end{tabular}

MEEM, mini exame do estado mental; Coh, coerência; O1-2, eletrodos occipitais.

\section{DISCUSSÃO}

Ao considerarmos os grupos estudados, obtivemos resultados estatisticamente consistentes entre os indivíduos normais e com provável DTA, resultados estes congruentes com a literatura por nós pesquisada $6,10,11,15$

Por outro lado, ocorreram achados inter-individuais que colocariam indivíduos do grupo DTA como normais, como por exemplo o paciente MAM (Tabela 2), que apresentou a coerência para sub-banda alfa 1 com valor de 0,69 . Este tipo de oscilação inter-individual só foi detectada na subbanda alfa 1.

Este achado pode ocorrer pelo fato de não termos conhecimento de qual seria a coerência deste indivíduo antes do início da doença, que eventualmente poderia ter valores por volta de 0,75 , 
sendo o dado encontrado no presente estudo já demonstrativo de declínio das funções corticais, porém ainda não o suficiente para enquadrar o paciente nos valores do grupo 2. Seria necessário, portanto, não apenas compararmos os pacientes com grupos controles, mas também com exames evolutivos do próprio paciente, no intuito de detectarmos a evolução da doença.

\section{CONCLUSÃO}

Nossos estudos mostram que a análise de coerência do EEG, quando analisado conjuntamente com os testes cognitivos e laboratoriais já estabelecidos para os quadros demenciais, poderá ser importante ferramenta no auxílio diagnóstico desta patologia, bem como no seu estudo evolutivo, com exames seriados.

Para tanto necessitamos ampliar o universo de indivíduos analisados por este método, bem como aprofundar os estudos entre outras áreas de relação inter e intra-hemisféricas.

\section{REFERÊNCIAS}

1. Harmony T, Marosi E, Fernandez T, Bernal J, Rodriguez M, Reyes A. EEG coherences in patients with brain lesions. Intern J Neurosci 1994;74:203-226.

2. Lehmann D. Multichannel topography of human alpha fields. Eletroenceph Clin Neurophisyol 1971;31:439-449.

3. Duffy FH, Albert MS, Mcnulty G, Garvey AJ. Age differences in brain electrical activity of healthy subjects. Ann Neurol 1984;16:430-438

4. Duffy FH, Albert MS, Mcnulty G. Brain electrical activity in patients with presenile and senile dementia of Alzheimer type. Ann Neurol 1984;16:439-448.

5. Besthorn C, Förstl H, Geiger-Kabisch C, Sattel H, Gasser T. EEG coherence in Alzheimer disease. Electroenceph Clin Neurophysiol 1994;90:242-245.

6. Wada Y, Nanbu Y, Koshino Y, Yamaguchi N, Hashimoto T. Reduced interhemispheric EEG coherence in alzheimer disease: analysis during rest and photic stimulation. Alzheimer Dis Assoc Disord 1998;12:175-181.

7. Leuchter AF, Cook IA, Newton TF, et al. Regional differences in brain electrical activity in dementia: use of spectral power and spectral ratio measures. Electroenceph Clin Neurophysiol 1993;87:385-393.

8. Leuchter AF, Spar JE, Walter DO, Weiner H. Electroencephalographic spectra and coherence in the diagnosis of Alzheimer type and multi-infarct dementia. Arch Gen Psychiatry 1987;44:993-998.

9. Locatelli T, Cursi M, Liberati D, Franceschi M, Comi G. EEG coherence in Alzheimer disease. Electroenceph Clin Neurophysiol 1998;106:229-237.

10. Wada Y, Nanbu Y, Kikuchi M, Kochino Y, Hashimoto T, Yamaguchi N. Abnormal functional connectivity in alzheimer's disease: intrahemispheric eeg coherence during rest and photic stimulation. Eur Arch Psychiatry Clin Neurosci 1998;248:203-208.

11. Stevens A, Kircher T. Cognitive decline unlike normal aging is associated with alterations of EEG temporo-spatial characteristics. Eur Arch Psychiatry Clin Neurosci, 1998;248:259-266.

12. Dunkin JJ, Osato S, Leuchter AF. relationships between EEG coherence and neuropsychological tests in dementia. Clin Electroenceph 1995;26:47-59.

13. Dunkin JJ, Leuchter AF, Newton TF, Cook IA. Reduced EEG coherence in dementia: state or trait marker? Biol Psychiatry 1994:35:870-879.

14. Sloan EP, Fenton GW, Kennedy NS, Maclennan JM. Neurophysiology and spect cerebral blood fow patterns in dementia. Electroenceph Clin Neurophysiol 1994:91:163-170.

15. Szelies B, Grond M, Herholz K, Kessler J, Wullen T, Heiss WD. QEEG mapping and pet in Alzheimer disease. J Neurol Sci 1992;110:46-56.

16. Bertolucci PHF, Brucki SMD, Campacci SR, Juliano Y. O mini-exame do estado mental em uma população geral: impacto da escolaridade. Arq Neuropsiquiatr 1994;52:1-7.

17. Luccas FJC, Braga NIO, Fonseca LC, Frochtengarten ML. Recomendações para o registro e interpretação do mapeamento topográfico do eletrencefalograma (EEG) e potenciais evocados sensoriais (PES) parte I: aspectos gerais. Braz J Epilepsy Clin Neurophysiol 1996;2:175-182.

18. Luccas FJC, Anghinah R, Braga NIO, et al. Recomendações para o registro/interpretação do mapeamento topográfico do EEG e potenciais evocados. Arq Neuropsiquiatr 1999;57:132-146.

19. Nuwer MR, Comi G, Emerson R, et al. IFCN standards for digital recording of clinical EEG. Electroenceph Clin Neurophysiol 1998;106:259-261. 